\title{
A Practical Approach for Residency Programs to Revive Graduate Medical Education during the Novel Coronavirus Disease 2019 (COVID-19) Pandemic: A Battle Proven Concept
}

\author{
Yousif Al-Saiegh* \\ Department of Cardiology, Pennsylvania Hospital, University of Pennsylvania Health System (UPHS), Philadelphia, PA, USA.
}

Corresponding author: Yousif Al-Saiegh, Department of Cardiology, Pennsylvania Hospital, University of Pennsylvania Health System (UPHS), Philadelphia, PA, USA.

Received date: April 26, 2021; Accepted date: May 7, 2021; Published date: June 15, 2021

Citation: Yousif Al-Saiegh, (2021). A Practical Approach for Residency Programs to Revive Graduate Medical Education during the Novel Coronavirus Disease 2019 (COVID-19) Pandemic: A Battle Proven Concept. J Clinical Research and Reports, 8(3); DOI:10.31579/2690-1919/163

Copyright: (C) 2021, Yousif Al-Saiegh, This is an open access article distributed under the Creative Commons Attribution License, which permits unrestricted use, distribution, and reproduction in any medium, provided the original work is properly cited.

\section{Abstract \\ The Novel Coronavirus Disease 2019 (COVID-19) Pandemic impacted the educational environment of Internal Medicine residency programs tremendously, shifting the focus from continued medical education to being the first line of defense while taking care of patients with COVID-19. Our article discusses an approach to reestablish medical education in the midst of a pandemic. \\ Keywords: medical education; novel coronavirus disease; pandemic; physician-to-patient encounters; social distancing; mitigation strategies}

\section{Manuscript}

Since the beginning of the Novel Coronavirus Disease 2019 (COVID-19) Pandemic in early 2020 Internal Medicine residency programs in the USA were forced to shift their focus from a balanced mix of clinical practice and continued medical education to treating patients with COVID-19. During the pandemic, there was an increased emphasis placed on limiting the exposure of medical residents to their best abilities. To preserve the educational character of our Internal Medicine residency program, we developed a modified curriculum that continued medical education while incorporating effective mitigation strategies.

Internal Medicine houses a breadth of subspecialties that makes the field attractive to medical graduates but also imposes a challenge for residency programs to incorporate sufficient educational opportunities to cover all facets of this immense field to prepare trainees for independent practice. Protected allotted time, in the form of dedicated elective blocks during residency training, allows trainees to explore subspecialties. These are broadly implemented in most residency programs to ensure an individualized training experience where trainees can spend time strengthening their knowledge in a field of interest or target an area of weakness. Electives are a crucial part of residency training to offer personalized learning. During the pandemic, our institution was forced to halt electives, as non-essential clinical rotations were discontinued to limit the risk of infection to patients and residents. Even after the peak of the pandemic, there continued to be a significant paucity in offered electives by internal medicine subspecialties in order to decrease exposure to medical residents who were in recent contact with COVID-19 patients as part of the clinical duties. This change, while essential to limit exposure risk, is one that is made at the expense of practical medical education.
We, therefore, developed a new "virtual elective curriculum" to ensure that residents are still able to explore their interests during elective blocks while minimizing exposure risk and limiting the spread of COVID-19. The virtual elective curriculum is composed of an assortment of online courses that have been selected by the program leadership and were determined to meet our educational standards. The online courses offer a wide variety of topics including clinical epidemiology, antibiotic stewardship, patient safety specialization, transgender medicine for general medical providers. At the end of each online course, residents are asked to summarize the studied materials, identify the salient teaching points, and share their newly acquired knowledge with their co-residents as an additional active learning strategy. This strategy maximizes the benefit of the virtual elective where residents enhance their knowledge and learn how to succinctly summarize and teach around this topic to their co-residents.

Residents were also encouraged to participate in daily educational conferences. Daily teaching conferences are presented by residents and moderated by attending physicians. The format of these includes case presentations, journal club, mortality and morbidity, and clinicalpathological conferences. The new format of teaching conferences offers additional flexibility to residents as they can be attended in person while all participants remain socially distanced from each other, or can be accessed virtually via video conference software. Teaching sessions are also recorded and stored on a server that can be accessed by all internal medicine residents at a later time. Despite their virtual format, they are interactive, and asking questions, posing discussion points is encouraged. The video conference software allows for real-time polls, questions to be entered in a chat box, and virtual hand signs to promote interactive learning and to improve understanding of the discussed concept. 
Bedside teaching is perhaps the area of medical education that has been most affected by the COVID-19 pandemic. Bedside teaching is a valuable and irreplaceable part of the medical curriculum to develop history-taking skills and hone physical exam techniques. However, it relies heavily on being in close proximity to a patient with the rest of the treatment team and poses imaginably a larger safety risk than other forms of teaching. The solution that we established at our institution was to practice diligent use of personal protective equipment including face masks and eye protection at all times, rigorous hand hygiene, and selective social distancing. Patients are also required to wear masks, unless unable to do so. We also limit the patients seen during medical rounds by prioritizing patients with educational value to be seen by the whole medical team. Out of this team, only two members who are most involved in the patient's care break the distance barrier of fewer than six feet to perform a focused physical exam and to demonstrate interesting findings. The rest of the team enters the room but remains more than six feet distant from the patient. This change of practice requires rooms to be large enough and only occupied by one patient to respect social distancing. Our health system transitioned to single-patient rooms at the beginning of the COVID-19 pandemic to mitigate patient-to-patient spread and more distanced physician-to-patient encounters.

\section{Abbreviations:}

COVID-19: Coronavirus Disease 2019
This work is licensed under Creative Commons Attribution 4.0 License

DOI: $10.31579 / 2690-1919 / 163$

Ready to submit your research? Choose Auctores and benefit from:

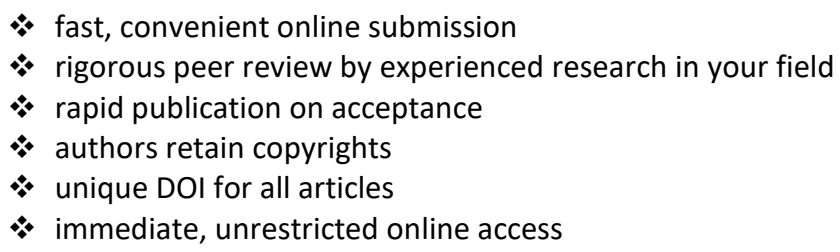

At Auctores, research is always in progress.

Learn more www.auctoresonline.org/journals/journal-of-clinicalresearch-and-reports 